\title{
Distributive and Dual Distributive Elements in Hyperlattices
}

\author{
R. Ameri, M. Amiri-Bideshki, S. Hoskova-Mayerova and A. B. \\ Saeid
}

\begin{abstract}
In this paper we introduce and study distributive elements, dual distributive elements in hyperlattices, and prove that these elements forms $\wedge$-semi lattice and $\vee$-semi hyperlattice, respectively. We use the properties of prime ideals and prime filters in hyperlattices. We will proceed to introduce the notion of dual distributive hyperlattices, $I$-filters and filters generated by dual distributive elements. Finally, the relationship between good homomorphisms and distributive (resp. dual distributive)elements in hyperlattices are investigated.
\end{abstract}

\section{Introduction}

Hyperstructure theory was first initiated by Marty [15] in 1934 when he defined hypergroups and started to analyze their properties. Since there are extensive applications in many branches of mathematics and applied sciences, the theory of algebraic hyperstructures (or hypersystems) has nowadays become a well-established branch in algebraic theory and has been developed in various fields, for more details see $[1,6,7,11,14,19,18]$ and books e.g. [6, 23]. Some applications can be found also in e.g. in $[4,5,8,10,16,17]$. The theory of hyperlattices was introduced by Konstantinidou in 1977 [13]. Barghi considered the prime ideal theorem for distributive hyperlattices [20]. Koguep,

Key Words: Hyperlattice, Prime ideal, Prime filter, Distributive element, Dual distributive element, Homomorphism.

2010 Mathematics Subject Classification: Primary 15A24, 15A60; Secondary 65F10.

Received: 01.07.2016

Revised: 12.11.2016

Accepted: 22.11.2016 
Nkuimi, and Lele studied ideals and filters in hyperlattices [12]. Rasouli and Davvaz defined fundamental relation on a hyperlattice and obtained a lattice from a hyperlattice. Moreover, they defined a topology on the set of prime ideals of a distributive hyperlattice [21, 22]. Ameri, Amiri-Bideshki, Saeid, Hoskova-Mayerova studied prime filters of hyperlattices in [2].

In this paper, we continue and deepen the ideas from [2], in particular we introduce distributive (dual distributive) elements, dual distributive hyperlattices, and $I$-filter in dual distributive hyperlattices. We give examples of the distributive elements and dual distributive elements in hyperlattices and we show that these concepts are independent. Also, we study $\wedge$-homomorphisms and $\vee$-homomorphisms in hyperlattices and consider relationship between $(\vee)$ or $(\wedge)$ homomorphisms and distributive (dual distributive) elements in hyperlattices. Finally, we define good homomorphisms and we obtain some results of them.

\section{Preliminaries}

In this section we give some results of hyperstructures and mainly hyperlattices that we need to develop our paper.

Definition 2.1. [15] Let $H$ be a nonempty set and $P^{*}(H)$ denotes the set of all nonempty subsets of $H$. Maps $f: H \times H \longrightarrow P^{*}(H)$, are called hyperoperations.

Definition 2.2. [13] Let $L$ be a nonempty set, $\wedge$ - be a binary operation, and $\vee$ - be a hyperoperation on $L . L$ is called a hyperlattice if for all $a, b, c \in L$ the following conditions hold:

[(i) $] a \in a \vee a$, and $a \wedge a=a ; a \vee b=b \vee a$, and $a \wedge b=b \wedge a ; a \in$ $[a \wedge(a \vee b)] \cap[a \vee(a \wedge b)] ; a \vee(b \vee c)=(a \vee b) \vee c$, and $a \wedge(b \wedge c)=(a \wedge b) \wedge c$ $a \in a \vee b \Longrightarrow a \wedge b=b$.

Let $A, B \subseteq L$. Then define:

$$
\begin{gathered}
A \vee B=\bigcup\{a \vee b \mid a \in A, b \in B\} \\
A \wedge B=\{a \wedge b \mid a \in A, b \in B\} .
\end{gathered}
$$

Definition 2.3. [12] Let $L$ be a hyperlattice. $L$ is called bounded if there exist $0,1 \in L$ such that for all $a \in L, 0 \leq a \leq 1$. We say that 0 is the least element and 1 is the greatest element of $L$.

Definition 2.4. [20] Let $L$ be a hyperlattice and $I$ be a nonempty subset of L. $I$ is called anideal if the following conditions hold:

[(i)]If $a, b \in I$, then $a \vee b \subseteq I$; If $a \in I, b \leq a$, and $b \in L$, then $b \in I$. 
Ideal $I$ is called a prime ideal if $a \wedge b \in I$ then $a \in I$ or $b \in I$ for all $a, b \in L$.

Definition 2.5. [20] Let $L$ be a hyperlattice and $F$ be a nonempty subset of $L$. $F$ is called a filter if the following conditions hold:

[(i)]If $a, b \in F$, then $a \wedge b \in F$; If $a \in F, a \leq b$, and $b \in L$, then $b \in F$.

Filter $F$ is called prime filter if $(a \vee b) \cap F \neq \emptyset$, then $a \in F$ or $b \in F$ for all $a, b \in L . L$ is distributive if for all $a, b, c \in L: a \wedge(b \vee c)=(a \wedge b) \vee(a \wedge c)$.

An example of an distributive hyperlatice can be found e.g. at [2].

Theorem 2.6. [12] Let $L$ be a distributive hyperlattice. Then $0 \vee 0=0$.

Definition 2.7. [3] Let $L$ be a nonempty set. Then $L$ is called $\wedge-$ semilattice if for all $a, b, c \in L$ the following conditions hold:

$[(\mathrm{i})] a \wedge a=a ; a \wedge b=b \wedge a ; a \wedge(b \wedge c)=(a \wedge b) \wedge c$.

\section{$3 \quad$ Ideals and filters in hyperlattices}

In this section, some properties of hyperlattices are mentioned and the relationship between prime ideals and prime filters in hyperlattices is discussed. Also the definitions of $I$-filter and the filter generated by $a \in L$ are recalled. More details can be found in [2].

In the sequel, $L$ denotes a bounded hyperlattice.

In [2] was defined the order $\leq$ on $L$ by

$$
a \leq b \Longleftrightarrow b \in a \vee b \Longleftrightarrow a \wedge b=a .
$$

The binary relation $\leq$ is reflexive, antisymmetric, and transitive, thus $(L, \leq)$ is a poset and for all $a, b \in L$ the following conditions hold:

[(i)] $a \in a \vee 0 ; 1 \in a \vee 1$; If $a, b \neq 0$ and $a \wedge b=0$, then $a, b \notin a \vee b$.

Theorem 3.1. [2] If $P$ is a prime ideal of $L$, then $L \backslash P$ is a prime filter of $L$.

Also holds: If $P$ is a prime ideal of $L$, then $L \backslash P$ is a $\wedge-$ semilattice. Which means, that the converse of Theorem 3.1 holds too. Now, we recall the following theorem:

Theorem 3.2. [2] Let $F$ be a prime filter of $L$. Then $L \backslash F$ is a prime ideal of $L$.

Definition 3.3. [2] Let $L$ be a non-empty set and $\vee-$ be a hyperoperation on $L$. Then $L$ is called a $\vee-$ semi-hyperlattice if for all $a, b, c \in L$ the following conditions hold:

$[(\mathrm{i})] a \in a \vee a ; a \vee b=b \vee a ; a \vee(b \vee c)=(a \vee b) \vee c$ 
In this part we recall for the readers convenient the definition of $I$-filters and filters generated by an element $a$ in a type of hyperlattices that are called dual distributive.

Definition 3.4. [2] A hyperlattice $L$ is called quasi dual distributive if for all $a, b, c \in L$.

$$
a \vee(b \wedge c) \subseteq(a \vee b) \wedge(a \vee c)
$$

$L$ is called weak dual distributive if: $a \vee(b \wedge c) \cap[(a \vee b) \wedge(a \vee c)] \neq \emptyset$;

$L$ is called dual distributive if: $a \vee(b \wedge c)=(a \vee b) \wedge(a \vee c)$

Clearly a quasi dual distributive hyperlattice is weak dual distributive and a dual distributive hyperlattice is quasi distributive and weak dual distributive.

The hyperlattices such that are distributive and dual distributive, are called strongly distributive hyperlattices.

Lemma 3.5. [2] Let $L$ be a dual distributive hyperlattice and $a \in L$. We define $F_{a}=\{x \in L: x \neq 0,1 \in x \vee a\}$, then $F_{a}$ is a filter of $L$.

Theorem 3.6. [2] Let $L$ be a dual distributive hyperlattice and $I$ be a nonempty subset of L. Define $F_{I}=\{x \in L: x \neq 0,1 \in x \vee a, \forall a \in I\}$. Then

$[(\mathrm{i})]$

1. $F_{I}$ is a filter of $L$.

2. $F_{I}=\bigcap_{a \in I} F_{a}$.

3. $F_{a} \cap F_{b}=F_{a \wedge b}$.

4. If $A \subseteq B$, then $F_{B} \subseteq F_{A}$.

$F_{I}$ is called $I$-filter generated by $I$.

\section{Distributive and Dual Distributive Elements}

In this section, we introduce distributive and dual distributive elements in hyperlattices. Also, we study Homomorphisms and good homomorphisms in hyperlattices. Then we study the relationship between dual distributive elements and homomorphisms. Finally, we give some properties of good homomorphisms.

Assume $L, L_{1}$ and $L_{2}$ are bounded hyperlattices.

Definition 4.1. Let $a \in L$. Then:

[(i)] The element $a$ is called distributive, if $a \wedge(x \vee y)=(a \wedge x) \vee$ $(a \wedge y)$, The element $a$ is called dual distributive, if $a \vee(x \wedge y)=$ $(a \vee x) \wedge(a \vee y)$, for all $x, y \in L$. 
Example 4.2. Let $L$ be the hyperlattice in Example 1 [2]. Let $L=\{0, a, 1\}$, and $\wedge$ and $\vee$ are given with Table 1 . Then the element $a$ is distributive

\begin{tabular}{l|lll}
$\wedge$ & 0 & $a$ & 1 \\
\hline 0 & 0 & 0 & 0 \\
$\mathrm{a}$ & 0 & $\mathrm{a}$ & $\mathrm{a}$ \\
1 & 0 & $a$ & 1
\end{tabular}

(a)

\begin{tabular}{c|ccc}
$\vee$ & 0 & $\mathrm{a}$ & 1 \\
\hline 0 & $\{0, a, 1\}$ & $\{a, 1\}$ & $\{1\}$ \\
$\mathrm{a}$ & $\{\mathrm{a}, 1\}$ & $\{a\}$ & $\{1\}$ \\
1 & $\{1\}$ & $\{1\}$ & $\{1\}$
\end{tabular}

(b)

Table 1

element, but the element 0 is not distributive, since $0 \wedge(a \vee 1)=\{0\}$ and $(0 \wedge a) \vee(0 \wedge 1)=L$.

Corollary 4.3. The following conditions hold.

[(i) $] L$ is a distributive hyperlattice if and only if for all $a \in L, a$ is a distributive element. $L$ is a dual distributive hyperlattice if and only if for all $a \in L, a$ is a dual distributive element.

Let $A \subseteq L$. We say that $A$ is a distributive subset, if and only if for all $a \in A, a$ is a distributive element. Similarly, a dual distributive subset of $L$ is defined.

Theorem 4.4. Let $a, b \in L$. Then the following conditions hold:

[(i)] If $a$ and $b$ are distributive elements, then $a \wedge b$ so is. If $a$ and $b$ are dual distributive elements, then the subset $a \vee b$ is a dual distributive subset.

2. Proof. Suppose $x, y \in L$. (i) We have:

$$
\begin{aligned}
(a \wedge b) \wedge(x \vee y) & =a \wedge b \wedge(x \vee y)=a \wedge[(b \wedge x) \vee(b \wedge y)] \\
& =[a \wedge(b \wedge x)] \vee[a \wedge(b \wedge y)]=[(a \wedge b) \wedge x] \vee[(a \wedge b) \wedge y]
\end{aligned}
$$

(ii) We have

$$
\begin{aligned}
(a \vee b) \vee(x \wedge y) & =a \vee b \vee(x \wedge y)=a \vee[(b \vee x) \wedge(b \vee y)] \\
& =[a \vee(b \vee x)] \wedge[a \vee(b \vee y)]=[(a \vee b) \vee x] \wedge[(a \vee b) \vee y]
\end{aligned}
$$

Corollary 4.5. [(i)]

1. Let $D(L)$ denotes the set of all distributive elements of $L$. Then $D(L)$ is a $\wedge$ - semi lattice. 
2. Let $D D(L)$ denote the set of all dual distributive elements of $L$. Then $D D(L)$ is a $\vee$-semi-hyperlattice.

Theorem 4.6. Let $a$ be a dual distributive element of $L$. We define $F(a)=$ $\{x \in L: x \in x \vee a\}$, then $F(a)$ is the least filter of $L$ which contains the element $a$.

Proof. Suppose $x, y \in F(a)$, We show that $x \wedge y \in F(a)$. We have: $x \in x \vee a$ and $y \in y \vee a$ and the element $a$ is dual distributive. Hence $x \wedge y \in(x \vee a) \wedge(y \vee a)=$ $a \vee(x \wedge y)$, so $x \wedge y \in(x \wedge y) \vee a$. Thus $(x \wedge y) \in F(a)$. Now assume $b \leq x$ and $b \in F(a)$, we show that $x \in F(a)$. We have $b \in b \vee a$, and by Definition $3.1, a \leq b$. Now, we have $a \leq b$, and $b \leq x$. The binary relation $\leq$ is reflexive, antisymmetric, and transitive, thus $(L, \leq)$ is a poset, therefore $a \leq x$ that is implied $x \in x \vee a$, so $x \in F(a)$. So $F(a)$ is a filter of $L$. Now, we show that $F(a)$ is the least filter of $L$ which contains the element a. Assume $F$ is a filter of $L$ such that $a \in F$. We show that $F(a) \subseteq F$. Let $x \in F(a)$. So $x \in x \vee a$, by Definition 3.1, $a \leq x$. Since $F$ is a filter and $a \in F$, so $x \in F$. Therefore $F(a) \subseteq F$.

Corollary 4.7. $F(a)=\{x \in L: a \leq x\}=\bigcap\{F \mid F$ is a filter and $a \in F\}$.

Proposition 4.8. [(i)]

1. $F(0)=L$;

2. $F(1)=\{1\}$;

3. If $a \leq b$ and $a, b$ are two dual distributive elements, then $F(b) \subseteq$ $F(a)$.

Corollary 4.9. If $L$ is a dual distributive hyperlattice, then $F(a), F(b) \subseteq$ $F(a \wedge b)$, for all $a, b \in L$.

Let $a$ be a dual distributive element of hyperlattice $L$ and $F(a)$ denote the least filter generated by $a$. We give a corresponding between distributive elements and $\wedge$ - homomorphisms. Also, we assume $P(L)$ is the power set of hyperlattice $L$. We know that $(P(L), \cup, \cap)$ is a lattice and every lattice is a hyperlattice.

Definition 4.10. Let $L$ and $K$ be hyperlattices and $f: L \longrightarrow K$ is mapping. Then for all $x, y \in L$

$[(\mathrm{i})] f$ is called $\wedge-$ homomorphism if:

$$
f(x \wedge y)=f(x) \wedge f(y)
$$


$f$ is called $\vee-$ homomorphism if

$$
f(x \vee y) \subseteq f(x) \vee f(y),
$$

$f$ is called homomorphism if $f$ is a $\wedge-$ homomorphism and a $\vee$ - homomorphism. $f$ is called good homomorphism if the following statements hold;

$$
f(x \wedge y)=f(x) \wedge f(y)
$$

and

$$
f(x \vee y)=f(x) \vee f(y) .
$$

A map $f$ is called isomorphism if $f$ is good, one to one, and onto homomorphism.

Example 4.11. Let $L$ be the hyperlattice in the Example 4.2 and $f: L \longrightarrow L$ is defined by: $f(0)=0, f(a)=a$, and $f(1)=a$. Then $f$ is a homomorphism, but it is not good. Since $f(0 \vee a)=\{a\}$, and $f(0) \vee f(a)=\{a, 1\}$, so $f(0 \vee a) \subset$ $f(0) \vee f(a)$.

Lemma 4.12. Let $a \in L$. Define $f_{a}: L \longrightarrow P(L)$ by: $f_{a}(x)=x \vee a$, for all $x \in L$. Then $f_{a}$ is a $\wedge-$ homomorphism, if and only if $a$ is a dual distributive element.

3. Proof. Let $f_{a}$ be a $\wedge$ - homomorphism and $x, y \in L$. So $f_{a}(x \wedge y)=f_{a}(x) \wedge$ $f_{a}(y)$, therefore $a \vee(x \wedge y)=(a \vee x) \wedge(a \vee y)$. So by Definition 4.1 (1), the element $a$ is dual distributive.

Conversely, suppose $a$ is a dual distributive element. So $a \vee(x \wedge y)=$ $(a \vee x) \wedge(a \vee y)$, and it means that $f_{a}(x \wedge y)=f_{a}(x) \wedge f_{a}(y)$. Therefore, $f_{a}$ is $\mathrm{a} \wedge-$ homomorphism.

Theorem 4.13. The following conditions for hyperlattice $L$ are equivalent.

[(i)] For all $a \in L, f_{a}$ is a $\wedge$ - homomorphism, $L$ is a dual distributive hyperlattice.

2. Proof. By Lemma 4.12 and Corollary 4.3, (2) proof is obvious.

Lemma 4.14. Let $a \in L$. Then the map $g_{a}: L \longrightarrow P(L)$ that is defined by $g_{a}(x)=\{a \wedge x\}$ for all $x \in L$, is a good homomorphism, if and only if $a$ is a distributive element.

Proof. Suppose $g_{a}$ is a good homomorphism and $x, y \in L$. So $g_{a}(x \vee y)=$ $g_{a}(x) \vee g_{a}(y)$, therefore $a \wedge(x \vee y)=(a \wedge x) \vee(a \wedge y)$ and this means that $a$ is a distributive element. 
Conversely, suppose the element $a$ is distributive. So $a \wedge(x \vee y)=(a \wedge x) \vee(a \wedge y)$, and it implies that $g_{a}(x \vee y)=g_{a}(x) \vee g_{a}(y)$. Since $a \wedge a=a$, so:

$g_{a}(x \wedge y)=a \wedge(x \wedge y)=(a \wedge a) \wedge(x \wedge y)=(a \wedge x) \wedge(a \wedge y)=g_{a}(x) \wedge g_{a}(y)$.

So $g_{a}$ is a good homomorphism.

Theorem 4.15. The following conditions are equivalent.

[(i)]For all $a \in L, g_{a}$ that is defined in Lemma 4.14 is a good homomorphism. $L$ is a distributive hyperlattice.

2. Proof. The proof is obvious by Lemma 4.14 and Corollary 4.3 .

Corollary 4.16. Let a be an element of L. Then the following conditions on the element a are equivalent:

$[(\mathbf{i})] a$ is a distributive element. The mapping $f: L \longrightarrow P(F(a))$ that is defined by $f(x)=\{a \wedge x\}$, for all $x \in L$, is a good homomorphism.

For a good homomorphism $f$, define $\operatorname{Ker}(f)=\{x \mid f(x)=1\} \cdot \operatorname{Ker}(f)$ is called kernel of $f$.

Example 4.17. [(i)]

1. If $f: L \longrightarrow L$ is defined by $f(x)=1$ for all $x \in L$, then $f$ is a good homomorphism and $\operatorname{Ker}(f)=L$.

2. Let $L$ be the hyperlattice in [12], i.e.: Let $L=\{0, a, b, 1\}$ and $\wedge$ and $\vee$ are given with Table 2

\begin{tabular}{l|llll}
$\wedge$ & 0 & $a$ & $b$ & 1 \\
\hline 0 & 0 & 0 & 0 & 0 \\
$a$ & 0 & $a$ & 0 & $a$ \\
$b$ & 0 & 0 & $b$ & $b$ \\
1 & 0 & $a$ & $b$ & 1
\end{tabular}

(a)

\begin{tabular}{c|cccc}
$\vee$ & 0 & $a$ & $b$ & 1 \\
\hline 0 & $\{0\}$ & $\{a\}$ & $\{b\}$ & $\{1\}$ \\
$a$ & $\{a\}$ & $\{0, a\}$ & $\{1\}$ & $\{b, 1\}$ \\
$b$ & $\{b\}$ & $\{1\}$ & $\{0, b\}$ & $\{a, 1\}$ \\
1 & $\{1\}$ & $\{b, 1\}$ & $\{a, 1\}$ & $L$
\end{tabular}

(b)

Table 2

Then $(L, \vee, \wedge, 0,1)$ is a distributive hyperlattice. Let moreover $f: L \longrightarrow L$ be defined by:

$$
f(a)=b, f(b)=a, f(0)=0, f(1)=1,
$$

then $f$ is an isomorphism and $\operatorname{Ker}(f)=\{1\}$. 
Proposition 4.18. Let $f: L_{1} \longrightarrow L_{2}$ be a good homomorphism. Then the following conditions hold:

[(i)]If $\operatorname{Ker}(f) \neq \emptyset$, then $\operatorname{Ker}(f)$ is a filter of $L_{1}$. If $x \leq y$, then $f(x) \leq f(y)$. If $f$ is an epimorphism, then $\operatorname{Ker}(f) \neq \emptyset$ also, $f(1)=1$ and $f(0)=0$. If $f$ is an isomorphism, then $\operatorname{Ker}(f)=\{1\}$.

3. Proof. (i): Let $x, y \in \operatorname{Ker}(f)$, and $a \in L_{1}$ which $x \leq a$. We show that $x \wedge y \in \operatorname{Ker}(f)$ and $a \in \operatorname{Ker}(f)$. Since $x, y \in \operatorname{Ker}(f), f(x)=f(y)=1$. Therefore $f(x \wedge y)=f(x) \wedge f(y)=1 \wedge 1=1$ and it means that $(x \wedge y) \in \operatorname{Ker}(f)$. If $x \leq a$, then by Definition $3.1 a \in(a \vee x)$, so $f(a) \in f(a \vee x)=f(a) \vee f(x)$. Therefore, $f(a) \in f(a) \vee 1$. By Definition 3.1, $1 \leq f(a)$ and since 1 is the greatest element, $f(a)=1$ and it means that $a \in \operatorname{Ker}(f)$.

(ii): If $x \leq y$, then by Definition3.1, $y \in x \vee y$. So $f(y) \in f(x \vee y)=$ $f(x) \vee f(y)$ and it means that $f(x) \leq f(y)$.

(iii): Let $f$ be an epimorphism. So, there exists $t \in L_{1}$ such that $f(t)=1$. Hence $t \in \operatorname{Ker}(f)$. Thus $\operatorname{Ker}(f) \neq \emptyset$. Since $x \leq 1$ for all $x \in L_{1}$ by $(i i)$, $f(x) \leq f(1)$, therefore $f(t) \leq f(1)$. Since $f(t)=1$, so $1 \leq f(1)$. Thus $f(1)=1$ (since 1 is the greatest element). Now, we prove $f(0)=0$. Since $f$ is onto, therefore $t \in L_{1}$ such that $f(t)=0$. We have $0 \leq x$ For all $x \in L_{1}$, so by (ii) is implied that $f(0) \leq f(x)$, for all $x \in L_{1}$. Therefore $f(0) \leq f(t)=0$ and since 0 is the least element, so $f(0)=0$.

(iv): Let $f$ be an isomorphism. Since every isomorphism is an epimorphism, $f$ is an epimorphism. Thus by $(i i i), f(1)=1$. Now, we assume $x \in \operatorname{Ker}(f)$. So $f(x)=1=f(1)$. Since $F$ is also one to one, so $x=1$. Hence $\operatorname{Ker}(f)=\{1\}$.

If $f(x) \leq f(y)$, where $x \leq y$, then $f$ is called isotone(order preserving map). If $f$ is a good homomorphism, then by Proposition 4.18, it is an order preserving map. Distributivity(dual distributivity)is preserved by good homomorphisms.

Set $f^{-1}(F)=\left\{x \in L_{1} \mid f(x) \in F\right\}$.

Proposition 4.19. [(i)]

1. If $f$ is a isomorphism and $F$ is a filter of $L_{1}$, then $f(F)$ is a filter of $L_{2}$.

2. If $F$ is a filter of $L_{2}$, then $f^{-1}(F)$ is a filter of $L_{1}$.

3. Let $a \in L_{1}$. Then $f(F(a)) \subseteq F(f(a))$.

Proof. (i): Let $x, y \in f(F)$ and $c \in L_{2}$ such that $x \leq c$. So there exist $a, b \in F$, such that $f(a)=x$ and $f(b)=y$. It is obvious that $(x \wedge y) \in f(F)$. We only, 
show that $c \in f(F)$. Since $f$ is onto, so there exists $d \in L_{1}$ such that $f(d)=c$. By $x \leq c$, we have $x \wedge c=x$, also $f(a \wedge d)=f(a) \wedge f(d)=f(a)$. Since $f$ is one to one, $a \wedge d=a$. Hence $a \leq d$ and $a \in F$. Since $F$ is a filter, so $d \in F$. Thus $f(d) \in f(F)$ that implies $c \in f(F)$.

(ii): Let $x, y \in f^{-1}(F)$ and $a \in L_{1}$ such that $x \leq a$. We must show that $x \wedge y \in f^{-1}(F)$ and $a \in f^{-1}(F) . x, y \in f^{-1}(F)$ implies $f(x), f(y) \in F$. Since $F$ is a filter, so $f(x) \wedge f(y) \in F$. Hence $f(x \wedge y) \in F$. Therefore $x \wedge y \in f^{-1}(F)$. Since $x \leq a$ and $f$ is a order preserving map, so $f(x) \leq f(a)$ that implies $f(a) \in F$. Thus $a \in f^{-1}(F)$.

(iii): Let $x \in f(F(a))$. Then there exists a element $b \in F(a)$ such that $x=f(b)$ and $a \leqslant b$. Since $f$ is isotone, $f(a) \leqslant f(b)$. So $f(a) \leqslant x$ and it implies that $x \in x \vee f(a)$. Thus $x \in F(f(a))$.

Proposition 4.20. Let $f$ be onto good homomorphism. Then the following conditions hold:

[(i)]If $a \in L_{1}$ is a distributive (dual distributive) element, then $f(a)$ is a distributive (dual distributive) element of $L_{2}$. If $L_{1}$ is distributive (dual distributive) hyperlattice, then $L_{2}$ is so.

2. Proof. (i): Let $x, y \in L_{2}$. We show that $f(a) \wedge(x \vee y)=(f(a) \wedge x) \vee(f(a) \wedge y)$. Since $f$ is onto, so there exist $b, c \in L_{1}$, such that $f(b)=x$ and $f(c)=y$. Thus we have:

$$
\begin{aligned}
f(a) \wedge(x \vee y) & =f(a) \wedge(f(b) \vee f(c)) \\
& =f(a) \wedge(f(b \wedge c))=f(a \wedge(b \vee c)) \\
& =f((a \wedge b) \vee(a \wedge c))=f(a \wedge b) \vee f(a \wedge c) \\
& =[f(a) \wedge f(b)] \vee[f(a) \wedge f(c)] \\
& =(f(a) \wedge x) \vee(f(a) \wedge y) .
\end{aligned}
$$

So $f(a)$ is a distributive element of $L_{2}$. While $a$ is a dual distributive element, similarly it is proved that $f(a)$ is dual distributive element of $L_{2}$.

(ii): It is proved similar to part $(i)$.

\section{References}

[1] H. AGHABOZORGHI, I.CRISTEA, M. JAFARPOUR, On complementable semihypergroups, Comm. Algebra, 44(4) (2016), 1740-1753.

[2] R. AMERI, M. AMIRI-BIDESHKI, A. B. SAEID and S. HOSKOVAMAYEROVA, Prime filters of hyperlatices, An. Stiint. Univ. "Ovidius" Constanta Ser. Mat., 24(2), (2016), 15-26. 
[3] G. BIRKHOFF, Lattice Theory, American Mathematical Society Colloguium Publications $[M]$, Providence, Rhode Island, 1940.

[4] J. CHVALINA, S. HOSKOVA-MAYEROVA, A. DEGHAN NEZHAD, General actions of hypergroups and some applications, An. Stiint. Univ. "Ovidius" Constanta Ser. Mat., 21(1), (2013), 59-82.

[5] J. CHVALINA, S. HOSKOVA-MAYEROVA, On certain proximities and preorderings on the transposition hypergroups of linear first-order partial differential operators, An. Stiint. Univ. "Ovidius" Constanta Ser. Mat., 22(1), (2014), 85-103.

[6] P. CORSINI, V. LEOREANU, Applications of Hyperstructure Theory, Kluwer Academic Publishers, Dordrecht, Hardbound, 2003.

[7] I. CRISTEA, S. JANCIC-RASOVIC, Compositions Hyperrings, An. Stiint. Univ. "Ovidius" Constanta Ser. Mat., 21(2), (2013), 81-94.

[8] I. CRISTEA, Regularity of Intuitionistic Fuzzy Relations on Hypergroupoids, An. Stiint. Univ. "Ovidius" Constanta Ser. Mat., 22(1), (2014), 105-119.

[9] I. CRISTEA, S. HOSKOVA, Fuzzy pseudotopological hypergroupoids, Iran. J. Fuzzy Syst., 6(4), (2009), 11-19.

[10] K. HASILOVÁ, Iterative Method for Bandwidth Selection in Kernel Discriminant Analysis.In: 32nd International Conference Mathematical Methods in Economics MME2014. Olomouc: Palacký University, Olomouc, (2014), 263-268.

[11] S. HOSKOVA, Topological hypergroupoids, Comput. Math. Appl., 64(9)(2012), 2845-2849.

[12] B. B. N. KOGUEP, C. NKUIMI, C. LELE, On fuzzy ideals of hyperlattice, Internat. J. Algebra, 2(2008), 739-750.

[13] M. KONSTANTINIDOU, J. MITTAS, An introduction to the theory of hyperlattice, Math. Balcanica, 7(1977), 187-193.

[14] M. KRASNER, A class of hyperrings and hyperfields, Int. J. Math. and Math. Sci., 2(1983), 307-312.

[15] F. MARTY, Surene generalization de la notion de group, Eighth Congress Scandinaves, Stockholm, (1934), 45-49.

[16] M. NOROUZI, I. CRISTEA, A note on composition (m,n)-hyperrings, An. Stiint. Univ. "Ovidius" Constanta Ser. Mat., 25(2) (2017)

[17] M.Č. MIKUŠKOVÁ, M. SEDLAČÍK, The application of regression trees to modelling ozone concentration measured in selected regions, Mendel 2016, 241-248.

[18] M. NOVÁK, Some basic properties of EL-hyperstructures, European J. of Combin. 34(2013), 446-459. 
[19] M. NOVÁK, $n$-ary hyperstructures constructed from binary quasi-orderer semigroups, An. Stiint. Univ. "Ovidius" Constanta Ser. Mat., 22(3), (2014), 147-168.

[20] A. RAHNEMAI-BARGHI, The prime ideal theorem for distributive hyperlattices, Ital. J. Pure Appl. Math., 10(2010), 75-78.

[21] S. RASOULI, B. DAVVAZ, Lattice derived from hyperlattices, Comm. in Algebra, 38(2010), 2720-2737.

[22] S. RASOULI, B. DAVVAZ, Construction and spectral topology on hyperlattice, Mediterr. J. Math., 7(2010), 249-262.

[23] T. VOUGIOUKLIS, Hyperstructures and their Representations, Hadronic Press, Inc., 1994.

\section{Acknowledgements.}

The first author partially has been supported by the "Algebraic Hyperstructure Excellence, Tarbiat Modares University, Tehran, Iran" and "Research Center in Algebraic Hyperstructures and Fuzzy Mathematics, University of Mazandaran, Babolsar, Iran". The third author was supported within the project "VÝZKUMFVT (DZRO K-217)" supported by the Ministry of Defence, Czech Republic.

Reza Ameri,

Department of Mathematics, Tehran University,

Tehran, Iran.

Email: rameri@ut.ac.ir

M. Amiri-Bideshki,

Department of Mathematics,

Payame-Noor University, Tehran,

Tehran, Iran.

Email: amirimohsen61@yahoo.com

Sarka Hoskova-Mayerova,

Department of Mathematics and Physics,

University of Defence,

Kounicova 65, Brno, Czech Republic.

Email: sarka.mayerova@unob.cz

A. B. Saeid,

Dept. of Pure Mathematics, Faculty of Mathematics and Computer

Shahid Bahonar University of Kerman

Kerman, Iran.

Email: arsham@uk.ac.ir 